\title{
Calcinosis dystrophica - operative Therapiemöglichkeit am Unterschenkel
}

\author{
Possibilities of Surgical Therapy on the Lower Leg
}

Autoren

Institut
W. Lehnert, P. Hinz, S. Lutze

Klinik und Poliklinik für Hautkrankheiten und Abteilung für Unfall- und Wiederherstellungschirurgie der Ernst-Moritz-Arndt-Universität Greifswald
Bibliografie

DOI http://dx.doi.org/

$10.1055 / \mathrm{s}-0033-1344639$

Akt Dermatol 2013; 39: 449-452

(c) Georg Thieme Verlag KG

Stuttgart · New York

ISSN 0340-2541

\section{Korrespondenzadresse \\ Priv.-Doz. Dr. med. \\ Wolfgang Lehnert \\ Hautklinik und Poliklinik \\ Universitätsmedizin Greifswald \\ Ferdinand-Sauerbruchstraße 1 \\ 17475 Greifswald \\ Lehnert@uni-greifswald.de}

\section{Zusammenfassung \\ $\nabla$}

Es wird über einen 68-jährigen Mann berichtet, bei dem sich im Verlauf von drei Jahren am rechten Unterschenkel von distal nach proximal zunehmend praetibial bis handflächengroße, teils ulzerierte derbe Kalkplatten an Haut und Unterhaut gebildet hatten. Nach Exzision dieser kalkfesten Veränderungen der Haut und Subkutis wurde eine Spanung der Tibia und eine Unterfüt-

\section{Einleitung \\ $\nabla$}

Unter Calcinosis dystrophica (C. d.) wird die Einlagerung von Kalksalzen in krankhaft verändertes oder absterbendes Gewebe ohne nachweisbare Störungen des Kalzium- und Phosphorstoffwechsels verstanden. Förderlich für die Ausfällung der Kalksalze soll die Alkalose des geschädigten oder absterbenden Gewebes infolge verminderter $\mathrm{CO}_{2}-$ Spannung sein [1].

Die Ursachen der lokalen Verkalkungen sieht Aschoff [2] in dem Auftreten der Substanzen, die eine erhöhte Affinität zum Kalk besitzen (Fibrin, Kolloid, Hyalin), des Weiteren in Ernährungsstörungen in einem dem Knochen nahestehenden Gewebe und im Kalkgehalt des Blutes, der allein aber nicht für die Kalkablagerungen ausreiche. Beobachtet wird die C. d. im chronischen Granulationsgewebe (tuberkulöse Lymphome, Ulcus cruris venosum), im durchblutungsgestörten Gewebe (Perniosis), in gutartigen (Hämangiome, Lipome, Epithelioma calcificans) und bösartigen (Karzinom, Sarkom) Geschwülsten der Haut [2].

Weiterhin finden sich Kalkeinlagerungen im perichondritischen Gewebe der Ohrmuschel nach Perniosis und als Phlebolithen in der Wand variköser Venen [1].

Die verkalkten Haut-Unterhautgewebe ulzerieren leicht, stellen eine permanente Infektionsgefahr [3] dar und verursachen anhaltende Schmerzen. Da die Kalkplatten eine Stagnation der Wundhei- terung des Defektes durch Muskelanteile des Triceps surae (Soleus-Plastik) vorgenommen. Nach Wundgrundkonditionierung - forciert durch Vakuumtechnik - bildete sich ein ausreichender Granulationsgeweberasen. Defektverschluss mittels Spalthauttransplantation in Mesh-graft-Technik. Dadurch konnte der Verkalkungsprozess am verbliebenen rechten Bein unterbrochen werden. Es wird an die gute Bildung von Granulationsgewebe aus Muskel- und Knochengewebe erinnert.

lung bedingen, besteht die Indikation zur operativen Beseitigung der Kalkplatten mit nachfolgender Defektdeckung. Das taktisch-therapeutische Vorgehen soll an einem Beispiel verdeutlicht werden.

\section{Anamnese, 68-jähriger Mann \\ $\nabla$}

1987 Myokardinfarkt und Apoplex mit mittelgradiger spastischer Lähmung der rechten Körperhälfte.

2003 Oberschenkelamputation links infolge peripherer arterieller Verschlusskrankheit (paVK). Prothetische Versorgung und ständige internistische Behandlung.

2004 Bagatellverletzung am verbliebenen rechten Bein mit daumennagelgroßer, schmerzhafter Ulzeration am Unterschenkel. Ab Oktober 2004 in unserer ständigen hautchirurgischen Behandlung.

\section{Lokalbefund \\ $\nabla$}

Über der distalen Tibiakante rechts $4 \times 2 \mathrm{~cm}$ großer, fibrinbelegter Gewebedefekt mit Umgebungserythem und kalkharten Resistenzen im Wundgrund und an den Rändern, Druckschmerzhaftigkeit ( $\bullet$ Abb.2). 


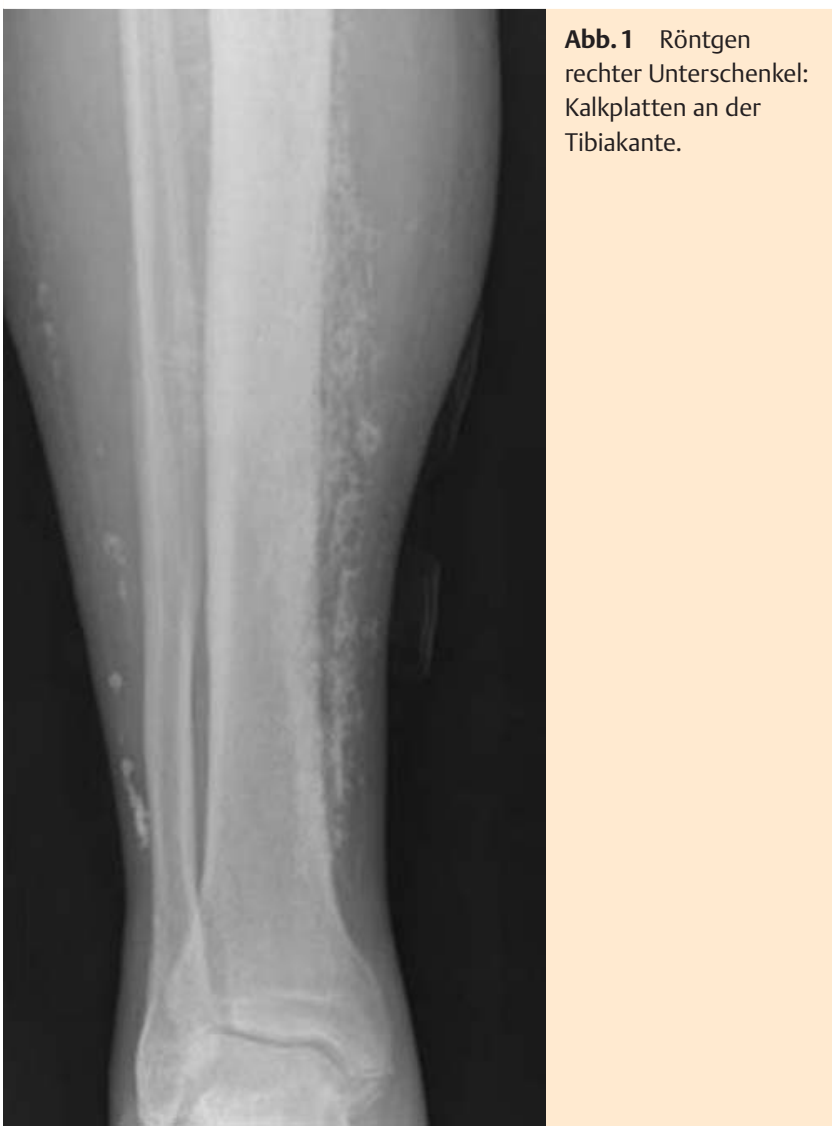

\section{Diagnostik}

$\nabla$

Duplexsonografie der peripheren Beckenund Beingefäße vom 18.7.2003

Ausgeprägtes postthrombotisches Syndrom beider Beine. Leitveneninsuffizienz der Vv. popliteae, rechts abschnittsweise parzielle Lumeneinengung der proximalen V. poplitea. Kalkeinlagerung der Becken- und Beinarterien.

\section{Weichteilröntgen rechter Unterschenkel vom 22.7.2004}

Im gesamten Verlauf der Tibiavorderkante und im distalen Beinabschnitt auch zwischen Tibia und Fibula sind ausgeprägte Verkalkungen nachweisbar, die der Knochenkante und dem Periost aufgelagert sind. Knöcherne Destruktionen sind nicht nachweis-

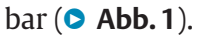

\section{Laboruntersuchungen vom 3.10.2004}

Im Normbereich $\mathrm{Ca}++$, Phosphat, Protein $\mathrm{C}$ und S. Fibrinogen $\mathrm{g} / \mathrm{l}$ 7,5 (1,8-3,5), CAP mg/L 8,1 $(<3,3)$, kein Nachweis von antinukleären Auto-Antikörpern.

\section{Becken-Bein-Arteriografie vom 7.10.2004}

1. Generalisierte Arteriosklerose mit Wandunregelmäßigkeiten.

2. Verschluss der A. femoralis communis links unmittelbar distal des Leistenbandes (Z.n. Oberschenkelamputation links).

3. Rechtes Bein: keine hämodynamisch wirksamen Obliterationen im Bereich der Arterien von Becken, Oberschenkel und Unterschenkel. A. tibialis posterior bis in Fußebene durchgängig, A. fibularis bis distalen Unterschenkel nachweisbar.

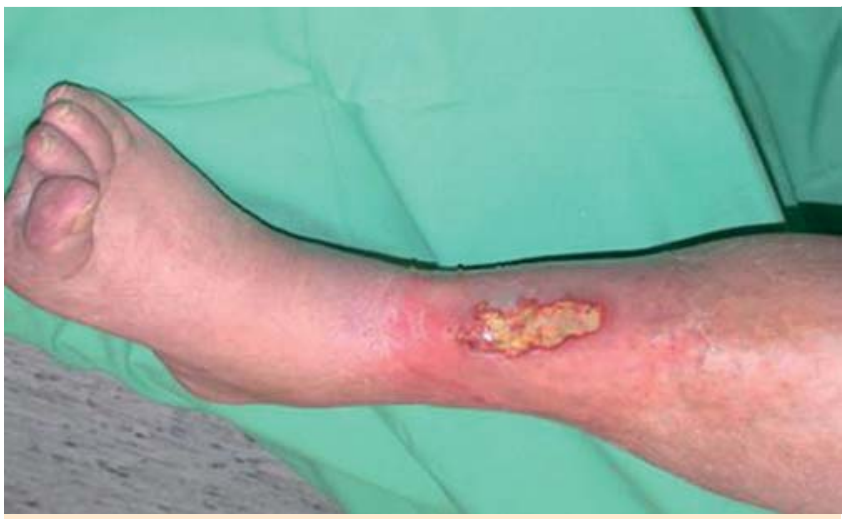

Abb.2 Ulzeration mit derben Kalkplatten im Wundgrund.

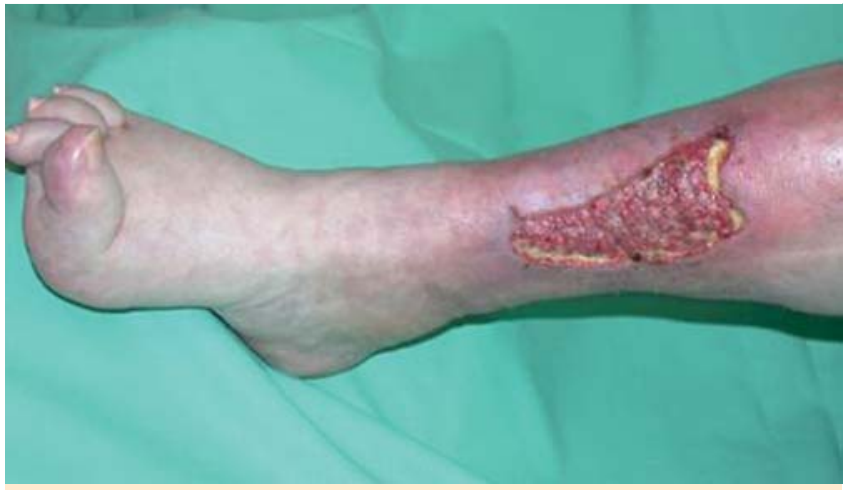

Abb. 3 Reverdin-Plastik: 4 Wochen postoperativ.

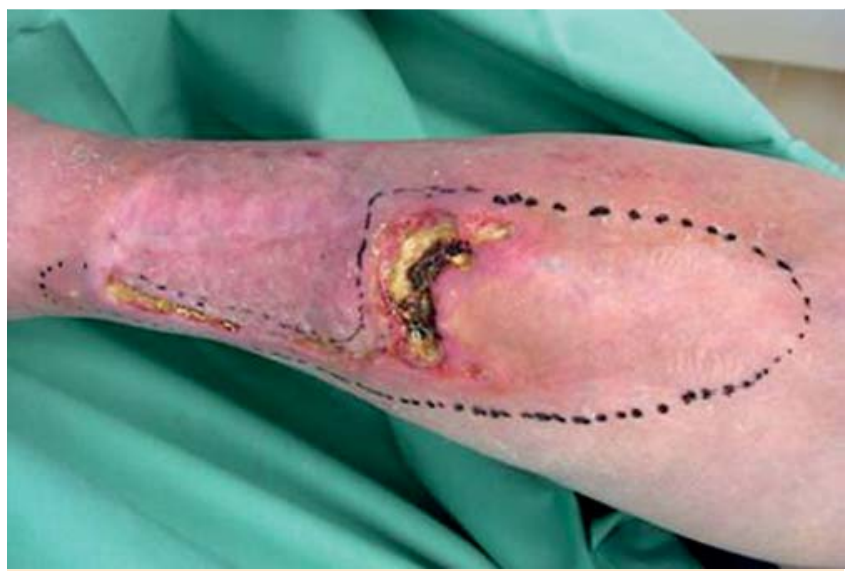

Abb.4 Ausgedehnte kutan-subkutane Verkalkungen. Präoperative Ausdehnung markiert.

MRT rechter Unterschenkel vom 17.3.2006

Ausgedehnte subkutane Verkalkungen der Haut und Unterhaut, fettige Degeneration des M. gastrocnemius und soleus. Kein Sequester, keine Fisteln.

Histologie (Nr. 1425/2004 vom 18.10.2004)

Histologisch zeigen sich korpuskuläre Deposite sowie größere Mengen polymorphkerniger Granulozyten. In den Arteriolen des subkutanen Fetts kein Kalziumphosphatnachweis. 


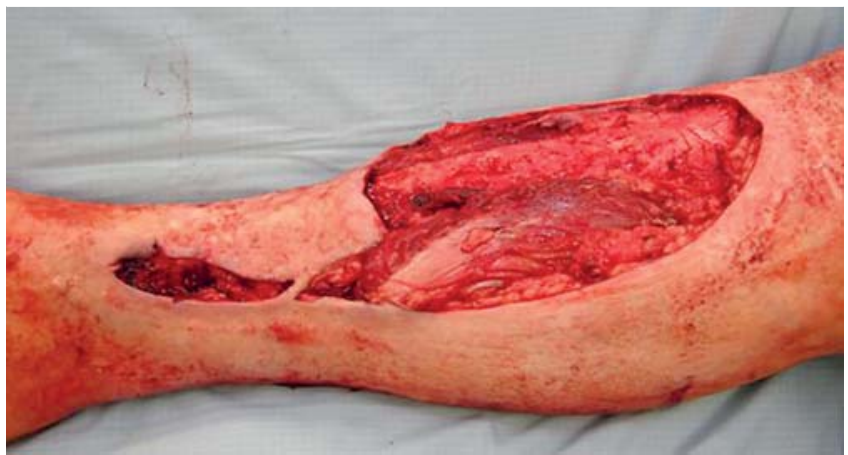

Abb.5 Rechter Unterschenkel: OP-Situs nach Entfernung großflächiger kutan-subkutaner Verkalkungen.

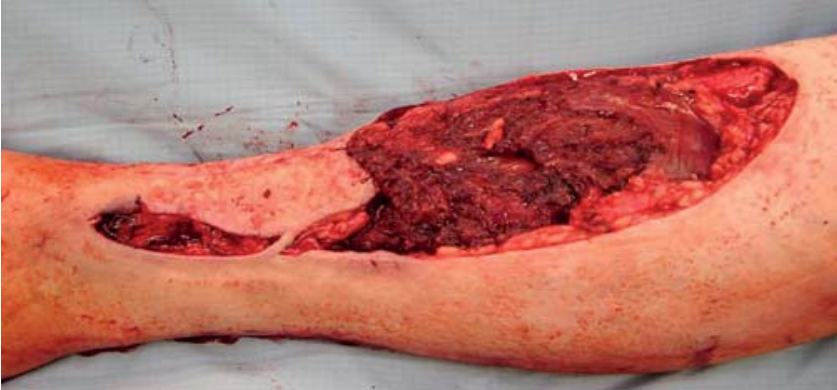

Abb.6 Rechter Unterschenkel: OP-Situs nach Soleusplastik und Spanung der Tibia.

\section{Therapie und Verlauf}

$\nabla$

Zunächst wurden am distalen rechten Unterschenkel in einer Ausdehnung von $7 \times 4 \mathrm{~cm}$ die Kalkspangen und Kalkplatten im Randbereich der nekrobiotischen Ulzeration und im Wundgrund entfernt; nach Wundgrundkonditionierung erfolgte eine Defektdeckung nach Reverdin [8] ( $\bullet$ Abb.3).

Die Wunde konnte dadurch bis auf randständige schmale Restdefekte verschlossen werden. Innerhalb eines Jahres breitete sich der Verkalkungsprozess jedoch weiter nach proximal aus: Die Haut-Unterhaut zeigte erneut druckdolente Ulzerationen mit derben, kalkharten Veränderungen mit Erythem in der Umgebung $(\bullet$ Abb.4).

Eine operative Behandlung musste daher die großflächige Entfernung der Kalkplatten am Unterschenkel mit nachfolgender Defektdeckung umfassen ( $\bullet$ Abb.5). Bekannt ist, dass aus Muskel- und Knochengewebe frisches Granulationsgewebe gebildet werden kann. Die Aa. surales (tibialis und fibularis) versorgen arteriell die Wadenmuskulatur, sie entspringen im oberen Kniegelenkbereich aus der A. poplitae [4]. Die Wadenmuskulatur ist nicht auf die in diesem Fall ohnehin schlechte arterielle Versorgung aus den 3 Unterschenkelarterien angewiesen.

Unter Berücksichtigung der schwach perfundierten A. fibularis lag es nahe, zur Defektdeckung Muskelanteile des M. triceps surae (M. gastrocnemius und M. soleus) in den Wundgrund als Basis für eine Spalthauttransplantation zu transponieren. Zusätzlich erfolgte dazu eine Spanung der Tibia ( $\bullet$ Abb.6). Die Wundgrundkonditionierung ( Abb.7) wurde durch Vakuumtechnik forciert und der Defekt durch Spalthauttransplantation in Mesh-graft-Technik (Dermatomeinstellung 0,3 mm, Carrier $1: 1,5)$ gedeckt ( $\bullet$ Abb.8).

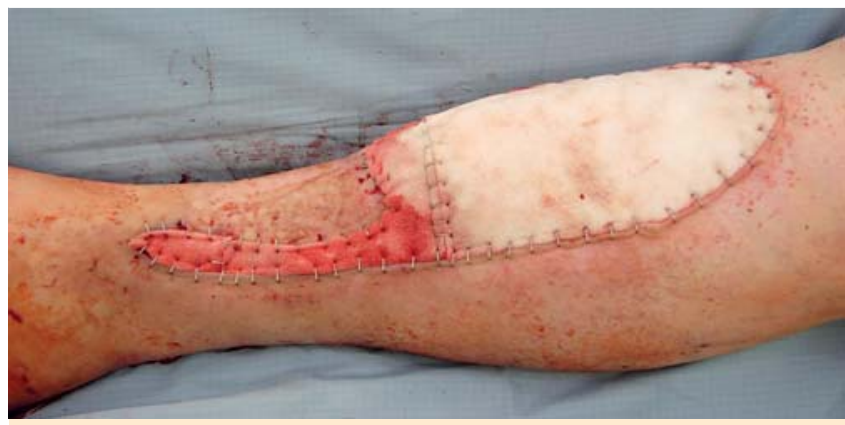

Abb.7 Wundabdeckung durch Polyethylenfolien zur Wundgrundkonditionierung.

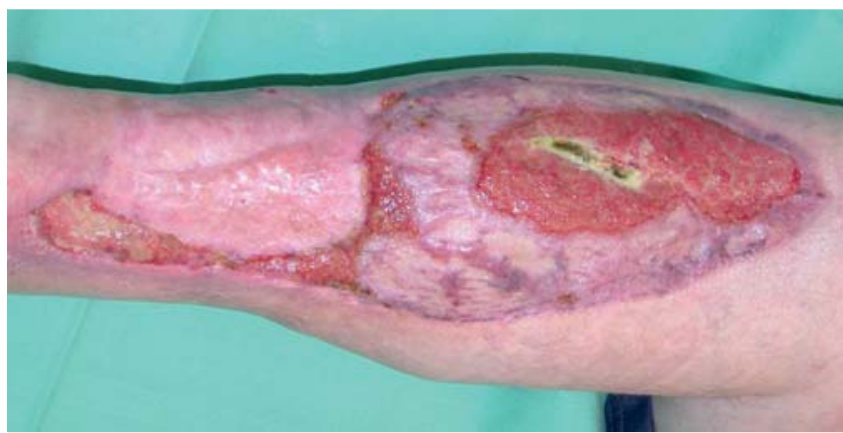

Abb. 8 Rechter Unterschenkel: 4 Wochen nach Spalthauttransplantation mit Restdefekten.

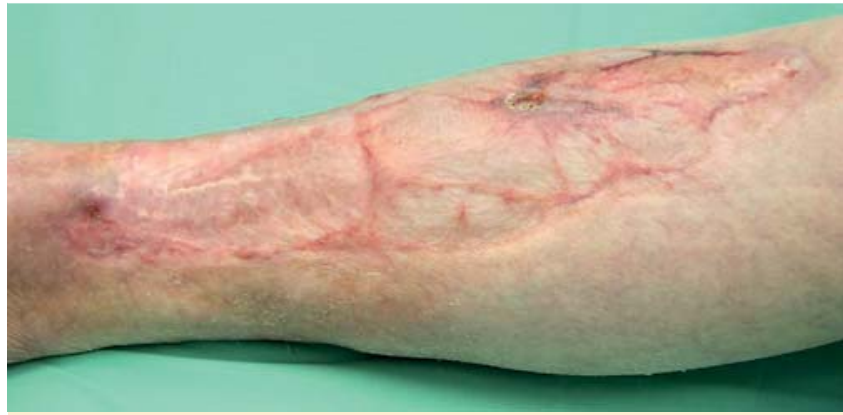

Abb.9 Rechter Unterschenkel: kompletter Defektverschluss nach erneuter Spalthauttransplantation der Restdefekte.

Der zentrale Anteil des Transplantates heilte nicht an, deshalb erfolgte eine erneute Spalthauttransplantation in gleicher Technik nach 2 Monaten mit dann vollständiger Abheilung der Defekte (๑ Abb.9)

Durch diese Maßnahme konnte nicht nur Schmerzfreiheit erzielt werden, sondern es konnten wirksame Gehübungen in Verbindung mit der Oberschenkelprothese links fortgeführt werden.

\section{Diskussion \\ $\nabla$}

Die Calcinosis dystrophica der Haut und Unterhaut wird im klinischen Alltag nicht selten diagnostiziert. Kürzlich konnten wir in 40 Jahre alten Verbrennungsnarben perkutane Kalkspieße feststellen. Wollina [5] fand bei 212 Patienten mit chronischen Unterschenkelwunden in 18\% Kalzifikationen ohne Assoziation mit einer Nierenerkrankung. Trotzdem ist die Kalziphylaxie bei allen chronischen, schmerzhaften Gewebedefekten in die Differenzial- 
diagnose mit einzubeziehen. Die Kalziphylaxie kommt nahezu in allen Fällen bei dialysepflichtigen Patienten vor (4\%). Die klinischen Symptome manifestieren sich zumeist als kutane Läsionen nach Ausbildung flächenhafter Erytheme, aus denen sich unterschiedlich konfigurierte Ulzerationen und Nekrosen bilden. Befallen sind in $90 \%$ die unteren Gliedmaßen. Aus den therapierefraktären Ulzerationen können Superinfektionen bis hin zur nekrotisierenden Fasziitis und Sepsis entstehen. Diagnostisch wertvoll sind letztendlich das klinische Bild und der histomorphologische Befund. Hier finden sich eine Mediaverkalkung und Intimaproliferation besonders an Arteriolen und kleinen Arterien der Haut und des subkutanen Fettgewebes.

Oft führt die Intimahyperplasie zu Okklusion des Gefäßlumens und begünstigt dadurch zusätzlich Thrombenbildung. Darüber hinaus können Epitheloidzellgranulome mit Riesenzellen vom Fremdkörper- und Langhanstyp im subkutanen Fettgewebe auftreten. Parallel zu den vaskulären Veränderungen findet sich oft eine kalzifizierende Pannikulitits im subkutanen Gewebe [3].

Vom klinischen Bild her sind auch Veränderungen der Granulomatosis disciformis chronica et progressiva Miescher differenzialdiagnostisch zu berücksichtigen. Prädilektionsstellen sind die Streckseiten der Unterschenkel, seltener Fußrücken und Beugeseiten; in $40 \%$ mit Diabetes mellitus vergesellschaftet. Kennzeichnend sind plattenartige Hautveränderungen, die an Sklerodermie erinnern, keine Kalkplatten aufweisen und selten schmerzhaft sind. Selten resultiert durch eine granulomatöse Entzündung Nekrobiose mit Ulzerationen [6]. Histologisch könnten Ablagerungen von IgM und IgA um die Gefäße auf eine Immunkomplexvaskulitis hinweisen [7].

Die chirurgische Entfernung der teilweise tief in das Weichteilgewebe reichenden Kalkplatten bei der Calcinosis dystrophica beseitigt vor allem die Gefahr schwerer Infektionen.

Reverdin-Hautläppchen [8] heilten in den Bereichen ein, die von Kalkplatten befreit waren. Eine nachhaltige Abheilung hängt vor allem vom Fortschreiten des Verkalkungsprozesses sowohl der großen als auch der kleinen Gefäße ab. In den größeren verkalkten Haut-Unterhautbereichen ist nach Entfernung aller klinisch nachweisbaren Verkalkungen und Defektdeckung mittels Muskeltransposition mit plastischem Hautverschluss vor allem die Infektionsgefahr gebannt und der erhebliche, permanente Schmerzzustand beseitigt. Ob dadurch der Prozess der Verkalkung beeinflusst werden kann, bleibt abzuwarten.

\section{Interessenkonflikt}

$\nabla$

Die Autoren geben an, dass kein Interessenkonflikt besteht.

Abstract

\section{Possibilities of Surgical Therapy on the Lower Leg $\nabla$}

A 68-year-old male patient is reported about. At the tibial area of the lower limb calcium deposits in form of sheets, plaques or fibers emerged, finally a painful necrobiotic non-healing ulcer formed. Renal failure, parathyroidism, autoimmune diseases were excluded. Arteriosclerosis without hemodynamically relevant obliterations was found. Removal of these calcium deposits induced the healing process, which was supported by transposition of parts of the soleus muscle and finally by skin mesh-grafts.

\section{Literatur}

1 Gertler W. Systematische Dermatologie. Leipzig: Verlag Ambrosius Barth; 1970

2 Kerl W. Beiträge zur Kenntnis der Verkalkungen der Haut. Archiv für Dermatologie und Syphilis 1919; 126: 172-251

3 Klode J, Klode D et al. Kalziphylaxie: Eine Übersicht. Vasomed 2006; 18: $6-12$

4 Platz F. Topographie und funktionelle Anatomie der arteriellen Hautversorgung am Unterschenkel: eine präparative angiologische Studie. In: Staubesand J, Schöpf E, Hrsg. Neue Aspekte der Sklerosierungstherapie. Berlin-Heidelberg - New York: Springer-Verlag; 1990: 105-130

5 Wollina U, Hasenröhrl K et al. Dystrophic Calcification in Chronic Leg Ulcers - A Clinico-pathologie Study. Dermatol. Surgery 2009; 35: 457-461

6 Miescher G, Leder M. Granulomatosis disciformis chronica et progressiva. Dermatologica 1948; 97: 25-34

7 Nost U, Jänner M, Schulz KH. Zur Entität der Necrobiosis maculosa. Granulomatosis disciformes chronica et progressiva Miescher und ihre Beziehung zur Necrobiosis lipoidica diabeticorum. Hautarzt 1974; 25: $325-332$

8 Reverdin JL. De la greffe épidermique. Arch Gén De méd 1869: 8 